\title{
Luminal A Breast Carcinoma
}

National Cancer Institute

\section{Source}

National Cancer Institute. Luminal A Breast Carcinoma. NCI Thesaurus. Code C53554.

A biologic subset of breast carcinoma defined by high expression of genes characteristic

of luminal epithelial cells, including estrogen receptor (ER), estrog en regulated protein

LIV-1, and the transcription factors hepatocyte nuclear factor 3, HNF3A, XBP1, and GATA

3. This subtype of breast cancer is associated with a good prognosis. 